\title{
Evaluation of a Trauma-Focused CBT Training Programme for IAPT services
}

\author{
Hannah Murray \\ Oxford Centre for Anxiety Disorders and Trauma, University of Oxford, \\ The Old Rectory, Paradise Square, Oxford OX1 1TW
}

\begin{abstract}
Background: Therapists in Improving Access to Psychological Therapies (IAPT) services are often expected to treat complex presentations of post-traumatic stress disorder (PTSD), such as individuals with multiple, prolonged or early life trauma histories and significant comorbidity, for which they have received minimal training. Although high recovery rates for PTSD have been demonstrated in randomized controlled trials, these are not always replicated in routine practice, suggesting that training interventions are required to fill the researchpractice gap. Aims: This study investigated the outcomes of a therapist training programme on treating PTSD with trauma-focused cognitive behavioural therapy (TF-CBT). Method: Twenty therapists from ten IAPT services participated in the training, which consisted of workshops, webinars and consultation sessions over a 6-month period. Results: Feedback indicated that participants found the training highly acceptable. PTSD knowledge and selfand supervisor-rated competence on TF-CBT measures improved following the training and improvements were maintained a year later. Client outcomes on a PTSD measure improved following the training. Participants reported attempts to disseminate learning from the course back to their teams. Conclusions: The findings indicate that the training programme was successful in improving TF-CBT knowledge, skills and outcomes for IAPT therapists. Tentative support for training 'trauma experts' within IAPT services was found, although institutional constraints and staff turnover may limit the sustainability of the model.
\end{abstract}

Keywords: Post-traumatic stress disorder, PTSD, training, CBT, IAPT.

\section{Introduction}

Training has an important role to play in the dissemination of evidence-based psychological therapies such as cognitive behavioural therapy (CBT). Whilst the development of effective treatments has advanced quickly over recent years, a 'research-practice gap' (McHugh and Barlow, 2010) runs the risk of leaving many clinicians insufficiently trained or supervised to deliver optimally effective treatments, at a time when the demand for psychological therapies is rapidly increasing (White, 2008).

The Improving Access to Psychological Therapies (IAPT) programme has trained thousands of therapists across the UK to deliver evidence-based psychological therapies

Correspondence to Hannah Murray, Oxford Centre for Anxiety Disorders and Trauma, University of Oxford, The Old Rectory, Paradise Square, Oxford OX1 1TW. E-mail: hannah.murray@psy.ox.ac.uk 
for common mental health problems. Outcome data from pilot sites showed clinical outcomes similar to those demonstrated in research studies (50-60\% recovery) and increased patient employment (Clark et al., 2009). However, outcomes differ across services and disorders. Recent figures for post-traumatic stress disorder (PTSD) suggest an average recovery rate of $37.8 \%$ (Health and Social Care Information Centre, 2016), but dropping as low as $15-20 \%$ for some services, making it one of the disorders with lowest recovery in IAPT.

One reason for poorer outcomes may be that clinicians treating PTSD often feel underconfident in applying some of the most effective techniques, such as imaginal reliving. Becker et al. (2004) found that only $17 \%$ of clinicians used imaginal exposure in treatment, with both lack of training and concerns about risks associated with the treatment cited as barriers. Gray et al. (2007) found positive attitudes towards evidence-based treatments for PTSD in their survey of trauma therapists, but these attitudes did not always correspond with behaviours; again barriers such as lack of time and access to training were cited as factors inhibiting practice of evidence-based therapies.

Furthermore, in an economic climate where NHS services are under increasing pressure, many IAPT services are treating increasingly complex cases, such as clients with PTSD to multiple events, prolonged and early life trauma, and additional complicating features such as co-morbid difficulties. IAPT services were originally intended for the treatment of mild to moderate psychological problems, and diploma-level CBT training courses may not cover interventions for more complex presentations. Although IAPT therapists have varying professional backgrounds and may have extensive experience of CBT, others have only undergone a year-long course, involving training and supervised practice in the treatment of all anxiety disorders and depression (see https://www.england.nhs.uk/ mental-health/adults/iapt/workforce/).

Studies have indicated that PTSD treatments are disseminable. Foa et al. (2005) trained therapists in both an academic treatment centre and a community clinic to high levels of competence in prolonged exposure and cognitive restructuring for PTSD, and clients showed significant improvements compared with a waitlist sample. Gillespie et al. (2002) reported an effective dissemination study whereby therapists treating survivors of the Omagh bombing were trained in cognitive therapy for PTSD and achieved excellent recovery rates, comparable to those in the research clinic samples of the same treatment (Ehlers et al., 2003, 2005). Several large-scale PTSD dissemination projects are ongoing. For example, the Veterans Health Administration in the USA is training thousands of clinicians in cognitive-processing therapy (Resick and Schnicke, 1992) and prolonged exposure (Foa et al., 2007), via workshops and consultation calls. Preliminary results suggest that the training has been widely accessed, and client outcomes have improved for both treatments (Karlin et al., 2010).

The training package evaluated in this report aimed to pilot a novel model for IAPT services: the training of 'trauma experts' across a region to provide expertise and supervisory capacity within their teams, and to disseminate knowledge. Such a model draws on 'cascade' or 'train the trainer' concepts, which have been recommended to encourage efficient dissemination (Becker and Stirman, 2011; Nakamura et al., 2014).

The training was evaluated at each level of Kirkpatrick's $(1967,1975)$ hierarchy of training evaluation: reactions, learning or knowledge acquisition, behaviour change and results, as 
well as penetration (whether trainees had successfully disseminated information and skills back to their services), and sustainability (Proctor et al., 2011).

\section{Method}

\section{The training course}

The training was delivered by the author, an experienced PTSD therapist, supervisor and trainer, and took place over a 6-month period between September 2013 and March 2014. It comprised 4 days of workshops, four half-day webinars, designed for the course, which were accessed remotely via an online videoconferencing system, and monthly consultation telephone calls (lasting an hour, in pairs) aimed at helping participants apply learning from the course to their current clients. The focus of the workshops was on learning and practising skills for assessing and treating PTSD using the Ehlers and Clark (2000) model of cognitive therapy. All participants were aware of the model from their CBT training, but had varied experience in its use. The initial workshop therefore focused on refreshing basic skills, such as imaginal reliving, working with hotspots and stimulus discrimination; later workshops focused on working with more complex issues, such as co-morbidity and multiple trauma. The webinars focused on modifications of the treatment for particular groups, including traumatic bereavement, military veterans, domestic abuse and childhood sexual abuse survivors. The training methods emphasized active learning using role-plays, therapy videos and experiential exercises. Overall, participants who attended all training events and consultation calls received 38 hours of training.

An online network was set up on the NHS Networks website (https://www.networks. nhs.uk/) to support the training. The network was used to promote communication between the trainer and trainees and to share resources such as papers, training slides and handouts. Message boards allowed the trainees to ask the trainer questions, or to communicate with each other.

\section{Context and participants}

The training was commissioned by a managed clinical network which supported the development of IAPT services across the East of England, a large region encompassing five NHS Trusts and ten IAPT services. Clinical leads for each IAPT service selected two 'step 3' therapists to participate in the training. Step 3 therapists have undergone CBT training, and see the more complex clients referred to IAPT. PTSD clients in IAPT are automatically seen at 'step 3', and should receive individual trauma-focused psychological therapy in line with NICE guidance (NICE, 2005).

The participants in the training course were therefore 20 CBT therapists working within IAPT. They had a range of professional backgrounds including clinical or counselling psychology, psychiatric nursing and social work, and had all completed additional CBT training. The course was predominantly female (19 females, 1 male).

Twenty therapists started the training course. One failed to complete the training, due to illness. The remainder completed the training, defined as missing no more than one training event. Six therapists were lost to follow-up. Of these, two were known to be off sick, and the others did not respond to attempts to contact. 


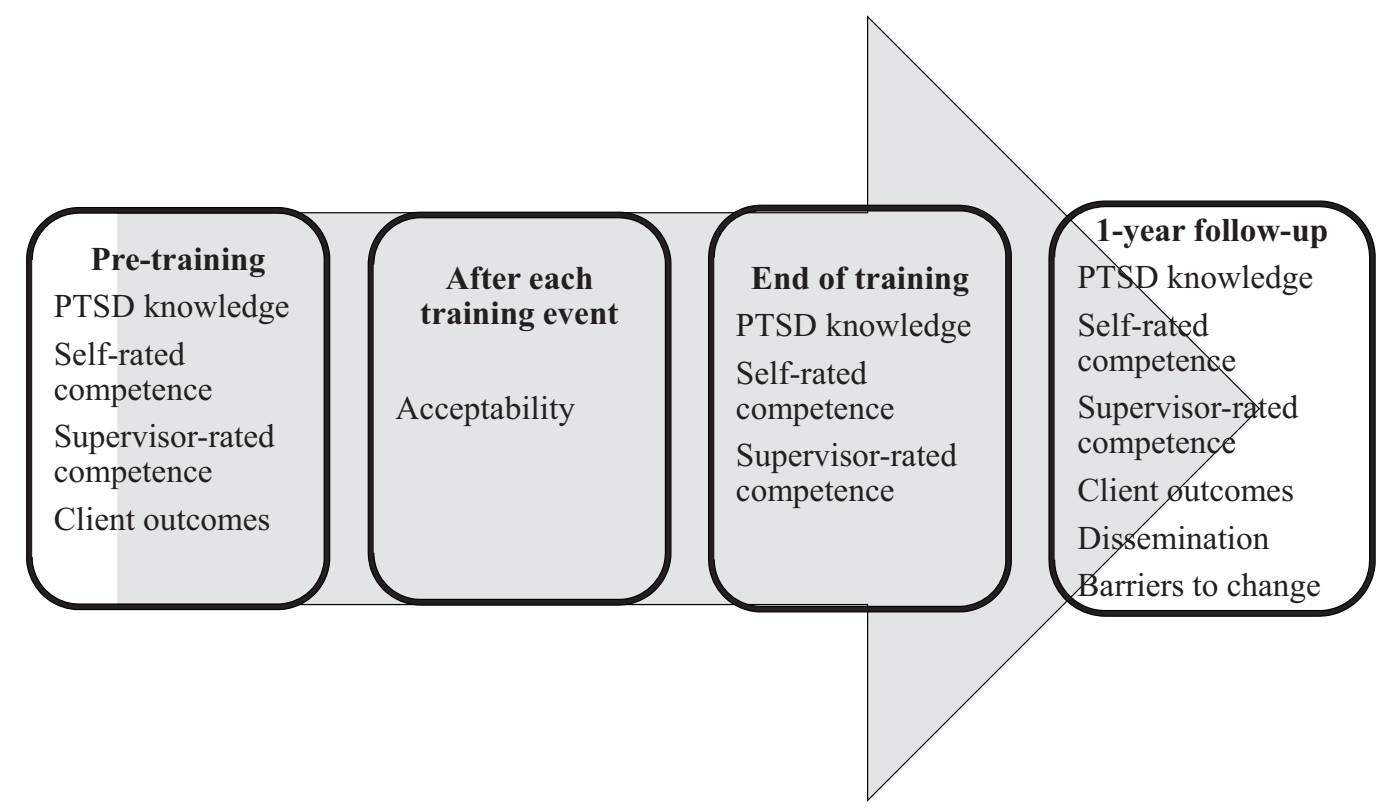

Figure 1. Data collection time points

\section{Evaluation and measures}

The training package was evaluated on a range of outcomes: acceptability, PTSD knowledge, self-rated competence, supervisor-rated competence and client outcomes. The time points for data collection are illustrated in Fig. 1.

Training acceptability. At the end of each training event, participants were asked to complete an anonymous feedback form. Free text items asked for comments on aspects of the event that trainees liked, and areas for improvement. Participants were asked to rate the event on variables using Likert scales, including whether the content of the workshop was at an appropriate level, relevant to their practice, well-structured and of a high quality.

PTSD knowledge. Participants completed a 'PTSD quiz' at the start and end of the training course, and a year later. The quizzes were different at each time point and were developed specifically for the training evaluation as no existing measure could be found. Potential quiz items were generated by a group of clinical psychologists specializing in PTSD and randomly assigned to pre-training, post-training or follow-up. The quiz items were chosen to measure basic knowledge about PTSD, such as prevalence, risk factors and diagnostic criteria.

Self-rated competence. Trainees completed a PTSD competence checklist at the start and end of the training programme and at 1-year follow-up. The checklist was adapted from Roth and Pilling's (2008) list of 68 knowledge, assessment and treatment competences for PTSD (Ehlers and Clark model) which was developed as part of a project by the Centre for Outcomes, Research and Effectiveness (CORE). The competence framework is available as a self-assessment tool on the CORE website (http://www.ucl.ac.uk/clinical-psychology/ CORE/competence_frameworks.htm), where participants rate themselves using a traffic light system, as 'I do not have this competence' (red); 'I have developed part of this competence' 
(amber); or 'I have fully developed this competence' (green). The competence list was adapted to a self-assessment form for the purpose of this study. This form has not been subject to any empirical validation.

Supervisor-rated competence. Trainees were asked to submit therapy tapes (audio or video-recorded) to their supervisors prior to the start of training (for a therapy session in the preceding 3 months), at the end of training, and a year later. The only instructions for tape selection were that it should show a CBT treatment session with a PTSD client, who had consented to recording.

Supervisors assessed the tapes using the CTS-PTSD; a version of the cognitive therapy scale (CTS; Young and Beck, 1980,1988) adapted to measure PTSD-specific competencies. The CTS-PTSD consists of 16 items, including standard items from the CTS measuring general CBT competences, with seven additional items relating to PTSD-specific skills. Competence on each item, and overall competence, is rated on a scale of 0 (poor) to 6 (excellent). Scores over 3 are generally considered 'competent', over 4 'proficient' and over 5 'expert'. Supervisors received no extra training in the use of the CTS-PTSD; however, they were familiar with the CTS, on which the measure is based, and were asked to contact the trainer if they had any difficulty with its administration.

Although the original CTS, and its revised version (Blackburn et al., 2001), have been widely used in research and clinical training, and have demonstrable levels of reliability and validity (e.g. Blackburn et al., 2001; Dimidjian et al., 2006), they measure general CBT skills, rather than the disorder-specific techniques that are often central to many treatments (Fairburn and Cooper, 2011). The CTS-PTSD was chosen for this study for increased sensitivity in assessing PTSD-specific skills. It is yet to be published or validated (A. Ehlers and S. Liness, unpublished observations, 2008).

Client outcomes. Clinical outcome data were collected for PTSD clients treated by participating therapists in the year before, and the year after, the training course. In IAPT services, clients routinely complete standardized measures, including the Patient Health Questionnaire-9 (PHQ-9; Kroenke et al., 2001), a 9-item measure of depression, and the Generalised Anxiety Disorder-7 (GAD-7; Spitzer et al., 2006), a 7-item measure of anxiety. Most services now also use disorder-specific measures, including the Impact of Events ScaleRevised (IES-R; Weiss and Marmar, 1997) for PTSD. Data on these measures were extracted from IAPT databases by participants and anonymized before analysis.

Barriers to change. At 1-year post-training, participants completed the 'Barriers to Change Questionnaire' (BARQ; Corrigan et al., 1992). This measure asks users to rate whether certain factors had impaired their capacity to apply training in the workplace on a scale of 0 (not a barrier at all) to 5 (insurmountable barrier). Sixteen potential barriers are rated, which are subcategorized into 'institutional constraints', 'support from colleagues', 'philosophical opposition', 'client dissatisfaction' and 'interference'.

The BARQ has a significant test-retest reliability $(r=0.41-0.92, P<0.01$; Corrigan et al., 1992) and has been used in other training evaluation studies to measure factors affecting training uptake (e.g. Myles and Milne, 2004; Kennedy-Merrick et al., 2008).

Dissemination. An idiosyncratic measure was developed for this study to assess attempts by participants to disseminate the training. Participants were asked whether they had changed their own practice as a result of the training, were providing supervision differently, or had 
provided training events or other resources to their team. Trainees were also asked to report any major changes to their team or role, for example if they had changed jobs.

\section{Analysis}

The primary analyses were conducted using multi-level modelling (MLM) software (MLwiN v2.32; Rasbash et al., 2009). This strategy was selected in order to account for withinparticipant correlation of responses recorded at a succession of time points. Models were developed for each evaluation outcome. Where responses were a proportion (e.g. a quiz score out of 10), a binomial logit model was applied. This was estimated initially using marginal quasi-likelihood (MQL), then refined using predictive quasi-likelihood (PQL) methods in order to limit downwards estimates caused by the small sample size, while minimizing convergence risk. Model fit was assessed using diagnostic plots supplemented by the ShapiroWilk normality test, in accordance with Collett (2003).

Significance testing was applied using one-sided tests, as it was not anticipated that the training would lead to a decrease in outcomes. In all cases the null hypothesis was of no change to scores.

\section{Results}

\section{Trainee satisfaction}

Overall, training events received high satisfaction ratings on the feedback measures, with an overall mean of 4.8/5 (SD 0.25), indicating that the training was acceptable. A one-way ANOVA showed no significant differences in overall satisfaction ratings across individual training events $(F=1.92 ; P=0.08)$.

A multi-level model was constructed to compare webinar and workshop ratings and revealed no significant differences $(z=-0.52 ; P=0.70)$, suggesting that participants found these formats equally acceptable.

Responses from the free text items on feedback questionnaires were categorized as positive or negative, and then subcategorized into comment type. The majority of comments were positive $(91.43 \%)$ and related primarily to training content and the style of delivery.

Attendance at training events and for consultation calls was consistently very high, and there were indications of high engagement with the course. For example, the content of consultation calls indicated that clients were applying techniques learnt in the workshops to clients on their caseload. Although not systematically recorded, the web-based elements of the course were less well attended, with fewer participants accessing the webinars compared with the workshops, and the online forum appeared to be used only sporadically.

\section{Knowledge of PTSD}

Mean scores for the PTSD quiz are illustrated in Table 1. The change in scores from pre- to post-training equates to an effect size of $r=0.60$, meeting Cohen's criterion of a large effect.

A multi-level model was built with participants as level 2 variables. Table 1 summarizes the estimates from fitting the logit model. There were significant improvements on quiz scores after the training, which were maintained at follow-up. Improvements from post-training to follow-up were non-significant. 


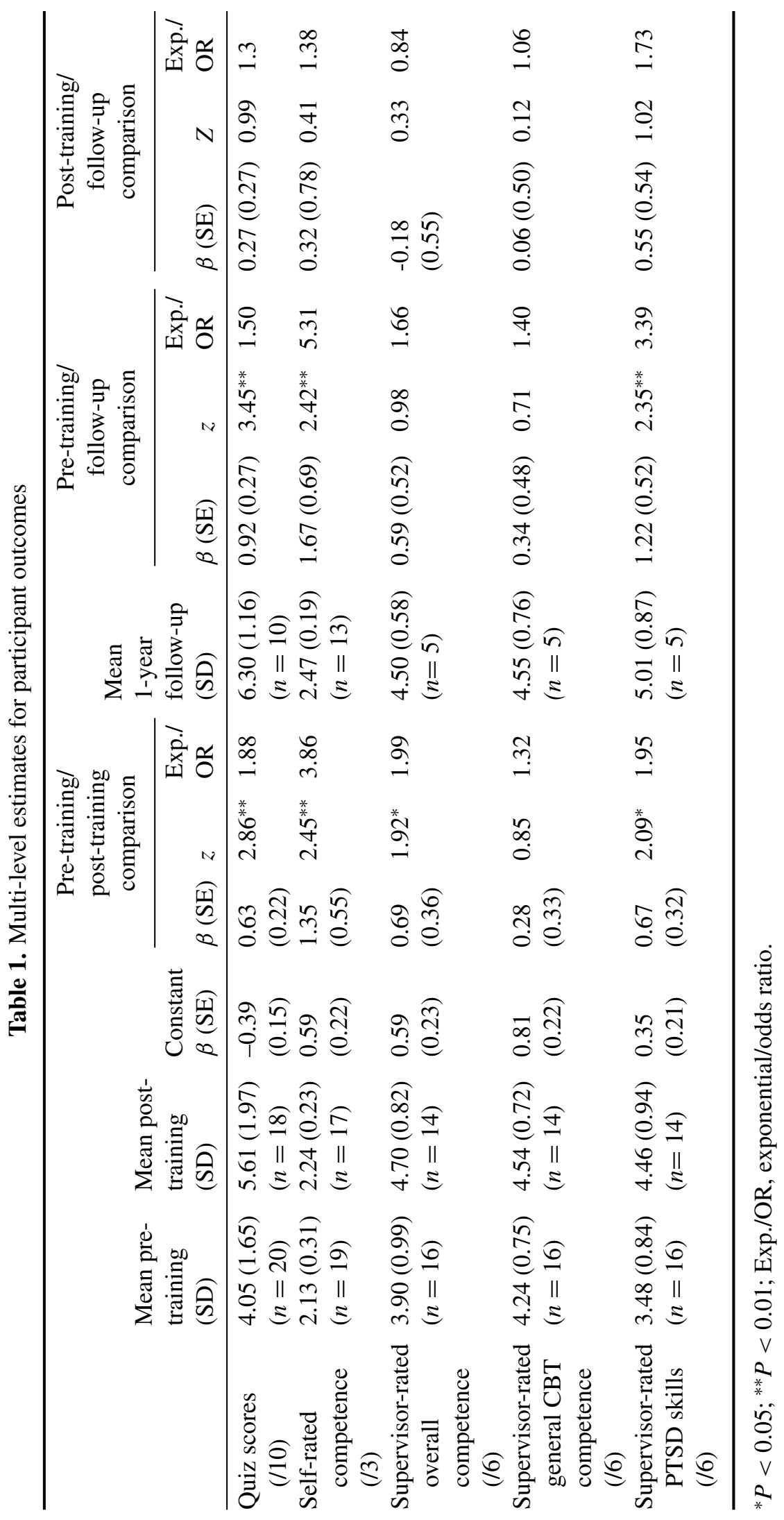




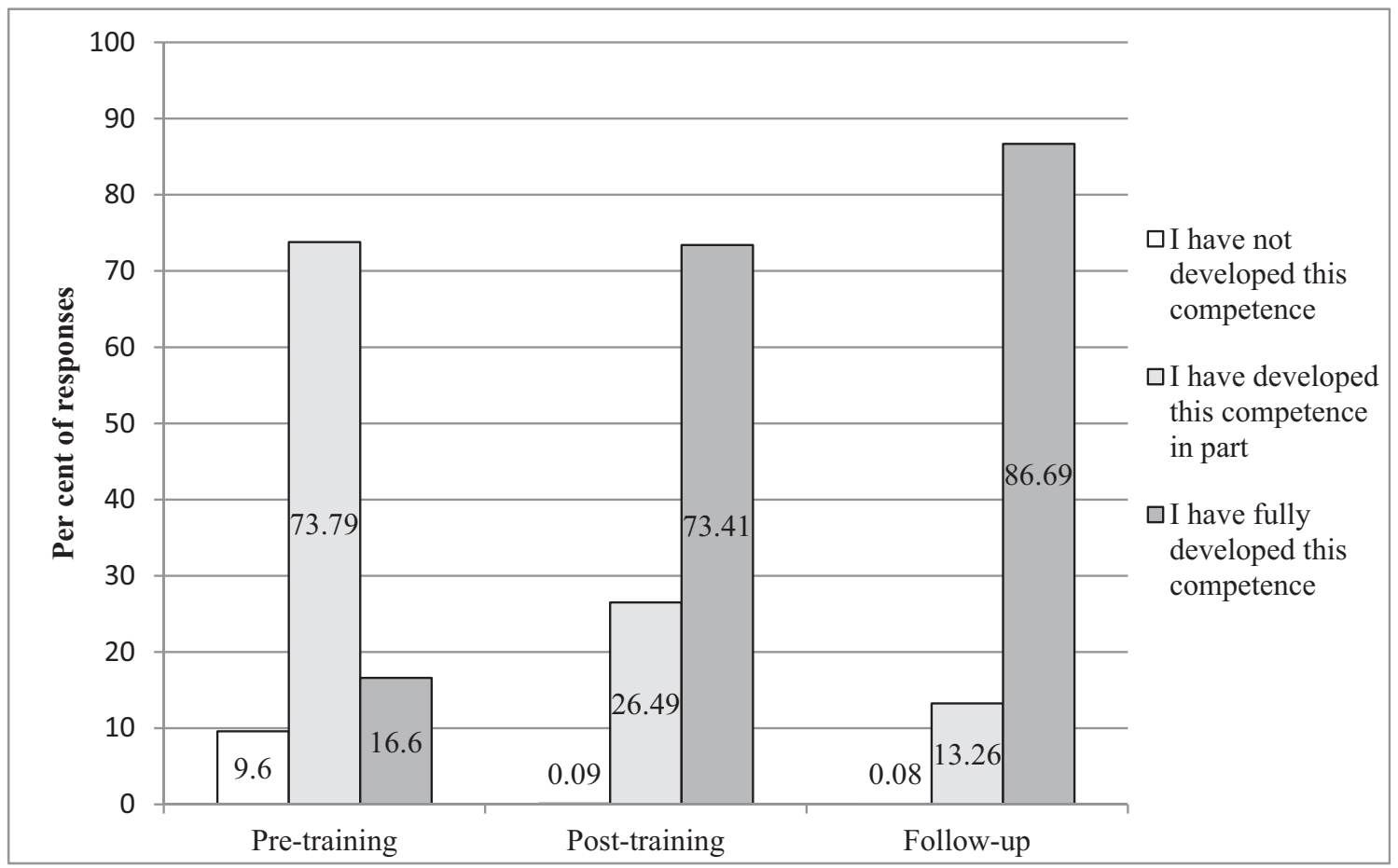

Figure 2. Self-competence ratings across time points

\section{Self-rated competence}

An overall self-rated competence score was calculated for each participant. Mean scores are summarized in Table 1. The change in scores from pre- to post-training equates to an effect size of $r=0.60$, meeting Cohen's criterion of a large effect.

A visual comparison for scores at the different time points is illustrated in Fig. 2.

A multi-level model was built with participants as level 2 variables. Table 1 summarizes the estimates from fitting the logit model. There were significant improvements on self-rated competence after the training, which were maintained at follow-up. Improvements from posttraining to follow-up were non-significant.

\section{Supervisor-rated competence}

Mean scores at each time point are summarized in Table 1. As well as the overall supervisor rating (out of six), a mean of scores on the generic CBT items (the nine items from the standard CTS) and the seven PTSD-specific items on the CTS-PTSD were calculated. Pretraining scores were in the 'proficient' range for generic CBT and 'competent' for PTSD skills. The training improved the PTSD-specific skills to the proficient level. The effect size for the improvement on PTSD-specific skills on the CTS-PTSD was high according to Cohen's classifications $(r=0.75)$.

Only five participants returned tapes at follow-up. No differences were found on quiz scores $(z=0.55 ; P=0.29)$, self-rated competence $(z=0.44 ; P=0.33)$ or CTS-PTSD ratings $(z=$ $-0.67 ; P=0.75)$ at post-treatment between those who did or did not submit tapes. 
A multi-level random effects model was built for each outcome, with participants at level 2. The results are summarized in Table 1.

Supervisors rated participants as significantly more competent on the CTS-PTSD overall following training, although this was not maintained at follow-up. When analysed by section, participants' scores on general CBT skills did not improve following training, but PTSDspecific skills significantly improved, and were maintained at follow-up.

\section{Client outcomes}

Scores on the PHQ-9 and GAD-7 at both pre- and post-treatment were available for 57 cases, and for 21 cases on the IES-R. A 3-level multi-level model was built, with clients at level 2 and participants at level 3 . Training time point (pre- or post-training) and treatment time point (pre- or post-treatment) were entered as predictor variables, with the key outcome relating to an interaction between the two. The outcome data models showed a poor diagnostic fit for a binomial model, probably due to correlation between the measured items, so a normal linear model was fitted. A folded log transformation was necessary to obtain acceptable normality and homoscedasticity.

The outcomes are summarized in Table 2. Overall, clients significantly improved on measures of PTSD, depression and anxiety following treatment. The improvement on the PTSD measure was significantly increased by the training intervention, i.e. scores on the IES dropped to a greater degree when therapists had undergone training. Scores for treatment completers were an extra 10 points lower on the IES when their therapist had completed the training. This effect was not found on measures of depression and anxiety.

\section{Dissemination}

Twelve participants reported their dissemination attempts at 1-year follow-up. No differences were found on quiz scores $(z=0.83 ; P=0.20)$, self-rated competence $(z=0.32 ; P=$ $0.37)$ or CTS-PTSD scores $(z=-0.09 ; P=0.53)$ between those who did or did not return questionnaires.

Participants were asked whether the training had changed their practice. On a scale of 0 (not at all) to 10 (completely), responses ranged from 7 to 10, with a mean of 8.33 (SD 0.98). Therapists were asked which areas of their work had changed as a result of the training. All reported having made changes to their own practice and all had made attempts to disseminate the training to their teams. These attempts included sharing new knowledge $(93.20 \%)$, sharing resources such as handouts $(84.60 \%)$, facilitating training events $(23.10 \%)$, changes to supervision (69.20\%), and taking on additional supervisory responsibilities (14.40\%).

On questions relating to significant changes to work environments since the course, two participants (16.67\%) were no longer working for the same service; one had left the NHS and another had moved to a different IAPT service. Four (20\%) of the participating services had changed provider, and two (10\%) therapists had changed role within their service.

\section{Barriers to change}

On the 'Barriers to Change Questionnaire', completed at follow-up, participants rated 'institutional constraints' as the greatest barriers to implementing the training in 


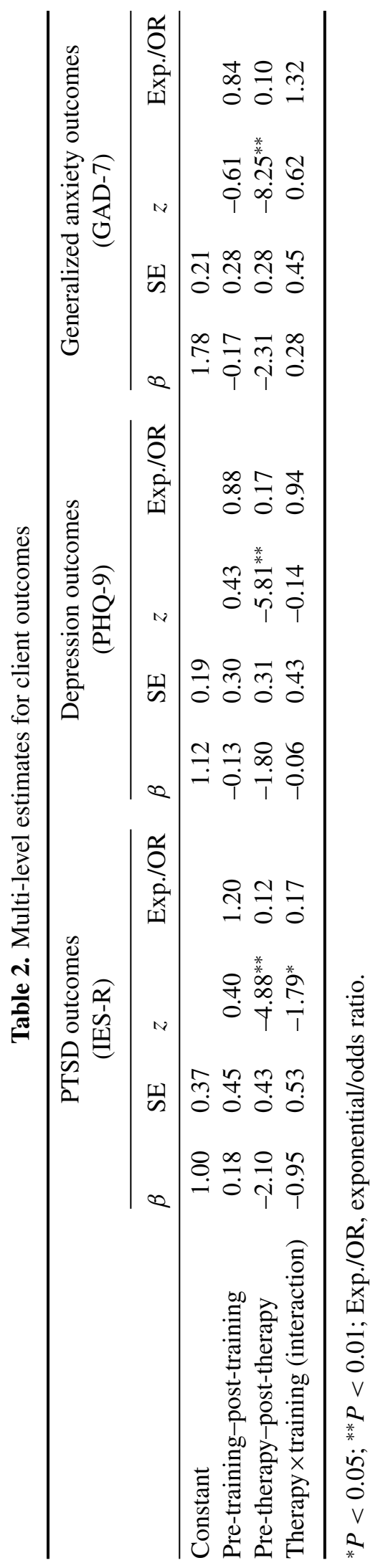


Table 3. Barriers to change, ranked

\begin{tabular}{lr}
\hline $\begin{array}{l}\text { Barrier to change (range: } 0-\text { not a barrier at all to 5 - insurmountable } \\
\text { barrier) }\end{array}$ & Mean (SD) \\
\hline Too many clients (institutional) & $2.25(1.36)$ \\
Too few staff (institutional) & $2.08(1.19)$ \\
Insufficient resources to help clients (institutional) & $2.08(1.24)$ \\
Limiting or restrictive practices in the way that client care is organized & $2.08(1.56)$ \\
$\quad$ (institutional) & \\
Other staff won't support me (support from colleagues) & $1.00(1.28)$ \\
Poor communication between staff (support from colleagues) & $0.92(0.79)$ \\
Staff turnover is excessive (support from colleagues) & $0.83(0.83)$ \\
Colleagues are not interested in applying for training (support from & $0.64(0.92)$ \\
$\quad$ colleagues) & \\
My clients do not understand the approach (client dissatisfaction) & $0.42(0.79)$ \\
Clients do not favour the approach (client dissatisfaction) & $0.42(0.79)$ \\
Relatives interfere with the programme (interference) & $0.25(0.45)$ \\
Family members or friends of the clients do not favour the approach (client & $0.08(0.29)$ \\
$\quad$ dissatisfaction) & \\
I don't believe that the training will work with clients (philosophical & $0.08(0.29)$ \\
$\quad$ opposition) & \\
Training is not appropriate for my clients (philosophical opposition) & \\
The approach is unethical (philosophical opposition) & $0.00(0.00)$ \\
Other clients interfere with the approach (interference) & $0.00(0.00)$ \\
\hline
\end{tabular}

their work place. Ratings of the barriers, ranked greatest to least, are displayed in Table 3.

\section{Discussion}

A training course in PTSD was found to be highly acceptable and to significantly improve the knowledge, self-rated competence and supervisor-rated competence (on PTSD skills) of a group of IAPT therapists. These improvements were maintained a year later. Outcomes of PTSD clients treated by the participating therapists were better following the training on a measure of PTSD, but not depression or anxiety. These findings suggest that a relatively brief training course in a disorder-specific treatment can significantly improve therapists' skills and outcomes.

Prior to the training intervention, therapists showed moderate levels of skill and confidence in treating PTSD. Generic CBT skills were scored as 'proficient' by supervisors but, prior to training, PTSD-specific skills were scored lower, although still in the 'competent' range. An expectation that therapists are proficient at treating the full range of psychological disorders seen in IAPT following a year-long CBT diploma course may be unreasonable. Therapists regularly encounter complicated cases in routine clinical practice and, unless changes are made to IAPT service inclusion criteria, disorder-specific training courses may be required to continue professional development post-qualification. 
Changes to client outcomes are rarely investigated in training evaluations. In this study, improvements were found on a PTSD outcome measure after the training intervention, but not on measures of depression or anxiety. Although PTSD was the main target of the training intervention, given the high rates of co-morbidity associated with the disorder, it is unclear why a change in depression and anxiety symptoms was not also found. Such changes may have become evident in a larger sample, or with longer follow-up of outcomes. It is also possible that, as participants were already competent CBT therapists, and were achieving good outcomes across all measures before the training, the additive factor of the training was finesse in delivering the trauma-specific elements of therapy which targeted PTSD symptoms in particular.

Effect sizes across the different outcome variables were broadly similar, although the different measurement methods used makes it difficult to draw conclusions about how the training impacted different outcomes. For example, a large change was seen in participants' self-assessed competency, indicating that they felt more confident in trauma-specific treatment competencies after the training. It cannot be established from the data whether the improved PTSD outcomes were related to participants' willingness to use trauma-focused techniques after training, or their improved competence in using elements of treatment which they were already delivering.

One aim of the training initiative was to trial a model whereby two 'trauma experts' were trained to provide a resource for the whole service. Although responses indicated that individuals did make attempts to pass on their learning to others in the team, there were several reasons for caution in concluding whether the model was successful. Within a year of the training, two therapists had left their service, three were off sick, and four services had new providers. Clearly, the model can only be sustainable if trained individuals remain embedded within teams and have sufficient resources and support to continue the specialist work.

In keeping with this, participants rated 'institutional constraints' such as high caseloads and too few staff as the main barriers to implementing the training. Even in the 'institutional constraints' category, ratings were generally in the mid-range, suggesting that such barriers were important, but not insurmountable. Very low ratings were found for most other perceived barriers. Both Myles and Milne (2004) and Kennedy-Merrick et al. (2008) also found institutional constraints to be the primary barriers in their studies of CBT training courses, and other studies have reported problems with the sustainability and penetration of training initiatives due to institutional problems (e.g. Schmidt and Taylor, 2002; Ebert et al., 2012).

\section{Limitations}

This evaluation was a naturalistic cohort study, and lacked randomization, control groups and blind assessment, which would be features of a controlled trial. While these are common limitations of training studies (Rakovshik and McManus, 2010), they reduce the generalizability of findings and conclusions. Without a control group, other unmeasured factors, such as access to other training and supervision, or service changes, may have contributed to improvements. However, the lack of improvement during the follow-up period, and on general CBT skills on the CTS-PTSD, appears contrary to this conclusion, and suggests that the programme succeeded in its aim of training PTSD-specific treatment 
skills. A further design limitation was the difficulty in dismantling the active components of the training. Workshops and webinars were equally popular, but the individual impact on learning was not measured, nor was the role of the consultation sessions [found to be integral in training transfer and durability in studies by Miller et al. (2004), Sholomskas et al. (2005) and Mannix et al. (2006)]. Although not measured, it was observable that the workshops and consultation calls were better attended than the web-based elements of the course. It may be that the online training led to a lower sense of accountability, a finding noted by McMillen et al. (2015) in their training study. Due to the absence of published measures, many of the outcomes in the study were measured using unvalidated tools. In particular, the knowledge quizzes were designed for the purpose of the study, and not psychometrically tested. Although randomization of items was used to promote equivalence of difficulty at each timepoint, this has not been confirmed. The sample size was small, and there was considerable missing data, particularly for supervisor-rated tapes at follow-up, which limits conclusions that can be drawn, and may have led to a reporting bias. However, no evidence was found of important differences between those who completed data and those who did not. Finally, the evaluation of dissemination was limited to self-reported attempts to disseminate, rather than an objective measure of these attempts.

\section{Conclusions}

Overall, the results seem to indicate that the trauma training package appeared to improve knowledge, skills and PTSD client outcomes for IAPT clinicians. The improvements were maintained a year later, and some evidence of attempts to disseminate learning back to teams.

This study could be extended in various ways. With respect to the limitations described, a randomized, controlled trial of the training package would allow firmer conclusions about its effectiveness to be drawn. Careful dismantling of the different elements of the training would allow for a better understanding of the effective ingredients. Longerterm and more intensive follow-up would allow the sustainability and penetration of the intervention to be fully assessed. Extending the evaluation of the dissemination of the programme, for example assessing broader benefits to teams, would be essential to evaluate the model.

Nevertheless, the results provide promising indications that relatively brief and costeffective training interventions can lead to service improvements, and may assist with closing the gap between research outcomes and routine clinical practice.

\section{Acknowledgements}

The author thanks Sarah Rakovshik, Sharif El-Leithy and Jo Billings for their valuable advice; Ruth Cocksedge for making the training possible; Glorianne Said for being a fantastic assistant; Dan Lunn for statistical support; and all the participating services and therapists.

Financial support: This research received no specific grant from any funding agency, commercial or not-for-profit sectors.

Conflicts of interest: None. 


\section{References}

Becker, K. D. and Stirman, S. W. (2011). The science of training in evidence-based treatments in the context of implementation programs: current status and prospects for the future. Administration and Policy in Mental Health and Mental Health Services Research, 38, 217-222.

Becker, C. B., Zayfert, C. and Anderson, E. (2004). A survey of psychologists' attitudes towards and utilization of exposure therapy for PTSD. Behaviour Research and Therapy, 42, 277-292.

Blackburn, I. M., James, I. A., Milne, D. L., Baker, C., Standart, S., Garland, A. and Reichelt, F. K. (2001). The revised cognitive therapy scale (CTS-R): psychometric properties. Behavioural and Cognitive Psychotherapy, 29, 431-446.

Clark, D. M., Layard, R., Smithies, R., Richards, D. A., Suckling, R. and Wright, B. (2009). Improving access to psychological therapy: initial evaluation of two UK demonstration sites. Behaviour Research and Therapy, 47, 910-920.

Collett, D. (2003). Modelling Binary Data, 2nd edn. Chapman and Hall/CRC.

Corrigan, P. W., Kwartarini, W. Y. and Pramana, W. (1992). Staff perception of barriers to behavior therapy at a psychiatric hospital. Behavior Modification, 16, 132-144.

Dimidjian, S., Hollon, S. D., Dobson, K. S., Schmaling, K. B., Kohlenberg, R. J., Addis, M. E. et al. (2006). Randomized trial of behavioral activation, cognitive therapy, and antidepressant medication in the acute treatment of adults with major depression. Journal of Consulting and Clinical Psychology, 74,658 .

Ebert, L., Amaya-Jackson, L., Markiewicz, J. and Fairbank, J. (2012). Development and application of the NCCTS learning collaborative model for the implementation of evidence-based child trauma treatment. In McHugh, R. K. and Barlow, D. H. (eds), Dissemination and Implementation of Evidence-Based Psychological Interventions, pp. 97-123. Oxford: Oxford University Press.

Ehlers, A. and Clark, D. M. (2000). A cognitive model of post-traumatic stress disorder. Behaviour Research and Therapy, 38, 319-345.

Ehlers, A., Clark, D. M., Hackmann, A., McManus, F. and Fennell, M. (2005). Cognitive therapy for post-traumatic stress disorder: development and evaluation. Behaviour Research and Therapy, 43, 413-431.

Ehlers, A., Clark, D. M., Hackmann, A., McManus, F., Fennell, M., Herbert, C. and Mayou, R. (2003). A randomized controlled trial of cognitive therapy, a self-help booklet, and repeated assessments as early interventions for posttraumatic stress disorder. Archives of General Psychiatry, $60,1024-1032$.

Fairburn, C. G. and Cooper, Z. (2011). Therapist competence, therapy quality, and therapist training. Behaviour Research and Therapy, 49, 373-378.

Foa, E. B., Hembree, E. A., Cahill, S. P., Rauch, S. A., Riggs, D. S., Feeny, N. C. and Yadin, E. (2005). Randomized trial of prolonged exposure for posttraumatic stress disorder with and without cognitive restructuring: outcome at academic and community clinics. Journal of Consulting and Clinical Psychology, 73, 953.

Foa, E., Hembree, E. and Rothbaum, B. O. (2007). Prolonged Exposure Therapy for PTSD: Emotional Processing of Traumatic Experiences Therapist Guide. Oxford: Oxford University Press.

Gillespie, K., Duffy, M., Hackmann, A. and Clark, D. M. (2002). Community based cognitive therapy in the treatment of post-traumatic stress disorder following the Omagh bomb. Behaviour Research and Therapy, 40, 345-357.

Gray, M. J., Elhai, J. D. and Schmidt, L. O. (2007). Trauma professionals' attitudes toward and utilization of evidence-based practices. Behavior Modification, 31, 732-748.

Health and Social Care Information Centre (2016). Psychological Therapies: Annual Report on the use of IAPT services - England, 2015-16. Available at: http://content.digital.nhs.uk/ catalogue/PUB22110 
Karlin, B. E., Ruzek, J. I., Chard, K. M., Eftekhari, A., Monson, C. M., Hembree, E. A. et al. (2010). Dissemination of evidence-based psychological treatments for posttraumatic stress disorder in the Veterans Health Administration. Journal of Traumatic Stress, 23, 663-673.

Kennedy-Merrick, S. J., Haarhoff, B., Stenhouse, L. M., Merrick, P. L. and Kazantzis, N. (2008). Training cognitive behavioural therapy practitioners in New Zealand: from University to clinical practice. New Zealand Journal of Psychology, 37, 8-18.

Kirkpatrick, D.L. (1967). Evaluation of training. In Craig, R. L. and Bittel, L. R. (eds), Training and Development Handbook, pp. 87-112. New York: McGraw-Hill

Kirkpatrick, D. L. (1975). Evaluating Training Programs. Tata McGraw-Hill Education.

Kroenke, K., Spitzer, R. L. and Williams, J. B. (2001). The Phq-9. Journal of General Internal Medicine, 16, 606-613.

Mannix, K. A., Blackburn, I. M., Garland, A., Gracie, J., Moorey, S., Reid, B. et al. (2006). Effectiveness of brief training in cognitive behaviour therapy techniques for palliative care practitioners. Palliative Medicine, 20, 579-584.

McHugh, R. K. and Barlow, D. H. (2010). The dissemination and implementation of evidence-based psychological treatments: a review of current efforts. American Psychologist, 65, 73.

McMillen, J. C., Hawley, K. M. and Proctor, E. K. (2015). Mental health clinicians' participation in web-based training for an evidence supported intervention: signs of encouragement and trouble ahead. Administration and Policy in Mental Health and Mental Health Services Research, 42, 1-12.

Miller, W. R., Yahne, C. E., Moyers, T. B., Martinez, J. and Pirritano, M. (2004). A randomized trial of methods to help clinicians learn motivational interviewing. Journal of Consulting and Clinical Psychology, 72, 1050-1062.

Myles, P. J. and Milne, D. L. (2004). Outcome evaluation of a brief shared learning programme in cognitive behavioural therapy. Behavioural and Cognitive Psychotherapy, 32, 177-188.

Nakamura, B. J., Selbo-Bruns, A., Okamura, K., Chang, J., Slavin, L. and Shimabukuro, S. (2014). Developing a systematic evaluation approach for training programs within a train-the-trainer model for youth cognitive behavior therapy. Behaviour Research and Therapy, 53, 10-19.

National Institute for Health and Clinical Excellence (NICE) (2005). Post-Traumatic Stress Disorder (PTSD): the management of adults and children in primary and secondary care. NICE clinical guideline 26. Available at: http://www.nice.org.uk/guidance/cg26

Proctor, E., Silmere, H., Raghavan, R., Hovmand, P., Aarons, G., Bunker, A. et al. (2011). Outcomes for implementation research: conceptual distinctions, measurement challenges, and research agenda. Administration and Policy in Mental Health and Mental Health Services Research, 38, 65-76.

Rakovshik, S. G. and McManus, F. (2010). Establishing evidence-based training in cognitive behavioral therapy: a review of current empirical findings and theoretical guidance. Clinical Psychology Review, 30, 496-516.

Rasbash, J., Charlton, C., Browne, W. J., Healy, M. and Cameron, B. (2009). MLwiN, version 2.1. Centre for Multilevel Modelling, University of Bristol.

Resick, P. A. and Schnicke, M. K. (1992). Cognitive processing therapy for sexual assault victims. Journal of Consulting and Clinical Psychology, 60, 748.

Roth, A. D. and Pilling, S. (2008). Using an evidence-based methodology to identify the competences required to deliver effective cognitive and behavioural therapy for depression and anxiety disorders. Behavioural and Cognitive Psychotherapy, 36, 129-147.

Schmidt, F. and Taylor, T. K. (2002). Putting empirically supported treatment into practice: Lessons learned in a children's mental health center. Professional Psychology: Research and Practice, 33, 483.

Sholomskas, D. E., Syracuse-Siewert, G., Rounsaville, B. J., Ball, S. A., Nuro, K. F. and Carroll, K. M. (2005). We don't train in vain: a dissemination trial of three strategies of training clinicians in cognitive-behavioral therapy. Journal of Consulting and Clinical Psychology, 73, 106. 
Spitzer, R. L., Kroenke, K., Williams, J. B. and Löwe, B. (2006). A brief measure for assessing generalized anxiety disorder: the GAD-7. Archives of Internal Medicine, 166, 1092-1097.

Weiss, D. S. and Marmar, C. R. (1997). The impact of event scale-revised. Assessing Psychological Trauma and PTSD, 2, 168-189.

White, J. (2008). CBT and the challenge of primary care: developing effective, efficient, equitable, acceptable and accessible services for common mental health problems. Journal of Public Mental Health, 7, 32-41.

Young, J. and Beck, A. T. (1980). Cognitive Therapy Rating Scale Manual. University of Pennsylvania, Philadelphia.

Young, J. E. and Beck, A. T. (1988). The Cognitive Therapy Scale (rev edn). Philadelphia, PA: Center for Cognitive Therapy. 